\title{
Towards a Capability-Based Decision Support System for a Manufacturing Shop
}

\author{
Reza Vatankhah Barenji \\ Department of Mechanical Engineering, Eastern Mediterranean University, \\ Famagusta, KKTC, Via 10 Mersin, Turkey \\ Reza.vatankhah@cc.emu.edu.tr
}

\begin{abstract}
To succeed, long term organizations must compete efficiently and out-perform their competitors in a dynamic environment. To survive in this competition, identification, classification and management of organizational capabilities are vital. This paper presents a methodology for developing a system to store, manage, and maintain intra-organizational capabilities for decision making processes on resources, processes and strategies for business opportunities. The proposed methodology is explored in an educational manufacturing cell.
\end{abstract}

Keywords: Capability, structural modeling, decision support system.

\section{Introduction}

The rapid growth of the global market encourages organizations to have a clear understanding of their area of expertise in order to maintain a competitive advantage. Some professionals and researchers refer to these areas of organizational expertise as capabilities [1]. Javidan, 1998[2] in his supplementary definition for core competency, believes that, the first step in successfully identifying and exploiting enterprises' core-competencies is creating a universal model for the competency, capability and organizational resources. Capability is seen as the enterprise's ability to exploit its resources. For better exploitation of the resources, information about activities which are realizable at the resource and the knowledge about how the resource and processes can work together are useful and essential issues.

Substantial improvements have been made on constructing decision support systems for the resources allocation process as well as on strategy selection regarding partnerships in the case of a new business opportunity for an organization or network of organizations. The existing decision support systems are found to be looking at resources and processes information separately [3]. The primary aim of this paper is to present a methodology for developing a system to store, manage and maintain intra-organizational capabilities used to develop a decision support system focusing on resources allocation and their related processes accompanied with a strategy selection for a new business opportunity of an enterprise. 


\section{Capability-based Decision Support System}

Capabilities refer to the company's ability to use its resources. Conceptually and empirically, Javidan's [2] capability definition is adopted by many of the researchers in this area. In manufacturing, a capability can be described as a set of information associated to the available resources and corresponding processes that could be performed by those resources, as well as the knowledge about how these resources and processes can be effectively, efficiently, and economically used [5].

The focus on capability as a key concept for decision making processes was first promoted in the managerial scientific literature with qualitative approaches of proficiency management [6]. However, since then, other scientific fields have integrated this significant notion with quantitative approaches, proposing decision support systems that brought up interesting results. A starting point for the integration of the concept of capability in the field of information and knowledge managerial science for decision making process has been proposed in [7], where the authors developed a model for manufacturing capability to support concurrent engineering. The formalization of the capability-related concepts covers different types of industrial decision processes. For instance, [8] proposed an approach for employing a capability model to support virtual enterprises; [9] proposed an approach to utilize a capability model to support global manufacturing co-ordination decisions; [5] developed a model to create and maintain knowledge for decision-making, where manufacturing strategy is described as knowledge related to processes and resources (knowledge model). Further research work on manufacturing capability and decision support systems focused on different contexts can be found at [9] [10].

Contribution to Collaborative Networks (CNs) - CNs can bring several benefits but their implementation needs many attractive and compelling essential sub functions that have to be clearly defined at different levels of abstractions. Capability modeling is a good starting point for responding many of these questions in this context, such as:

- What can organizations do?

- How can organizations exploit their resources?

- How to measure the organization's willingness to do collaboration?

- How can we boost VO and VBE performance?

- What is the comprehensive tool for partner selection in VO and VBE?

- What is the tool to do better collaboration?

Certainly many other relevant questions may be asked in relation to capability modeling in CNs context.

\section{$3 \quad$ Research Methodology}

The research methodology adopted in this work is sub-divided into three parts as shown in Fig. 1. Between "Preliminary study and problem definition phase" and "Design and development phase" there exists a "Verification block". The adopted modeling approach is based upon object-oriented analysis and design techniques. The Unified Modeling Language (UML) is employed as a graphical modeling language, 
which enables system developers, analyzers, and stakeholders to design and visualize the relevant concepts.

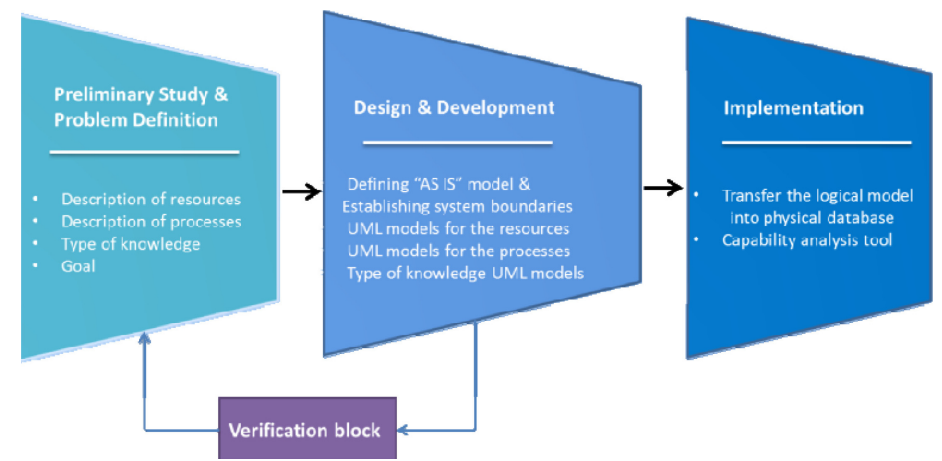

Fig. 1. Research Methodology

The "Verification block" allows to iterate between the "Preliminary study problem definition phase" and "Design and development phase". A capability management system is developed in the "Implementation phase" and an application architecture for the desired decision support system is proposed for the case of a shop floor. The proposed methodology is evaluated in case of the Computer Integrated Manufacturing (CIM) laboratory of the Eastern Mediterranean University (Fig. 2).

\section{$4 \quad$ Preliminary Study and Problem Definition Phase}

The EMU-CIM laboratory was designed for both educational and research purposes. The laboratory consists of three stations: Station 1 is a machine tending station, which holds a CNC milling machine and a five-axis vertically articulated robot designed to work in industrial training facilities. Station 2 is an assembly and quality control station, which has one robot. This robot has a pneumatic gripper and works in connection with the peripheral station devices such as a ball feeder, a gluing machine and a laser-scan micrometer device. Station 3 is an automatic storage and retrieval system (AS/RS), which contains 36 storage cells and a robot with the ability of taking and placing the work pieces. A conveyer integrates the 3 stations performing the material transport within the cell. The overall system is running under a supervisory host control consisting of a set of station IPC's, a PLC for controlling the conveyor and a host computer that allows management of the cell orders, employing the OPEN CIM software.

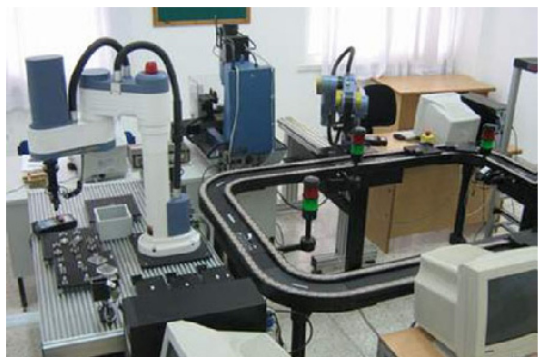

Fig. 2. CIM laboratory of Eastern Mediterranean University 
Several operations can be executed in the EMU-CIM Lab. For illustration, the assembling operation, deals with two work pieces; A and B. The system starts with a command from the host computer to the AS/RS for loading the work pieces A and B onto the conveyer. When the parts reach the "assembly and quality control" station, the station's robot takes the parts and puts them in the ball loading position; where four balls are loaded using the robot. At that time, the robot takes the sub-assembly and puts it into the assembling station. The gluing machine starts to work and injects the glue in the desired points on the sub-assembly, and then the robot places part B into the subassembly. The product returns to the AS/RS system via the conveyer and its associated station's robot.

\section{Design and Development Phase}

Defining "As Is' Model and Establishing System Boundaries. The design and development phase started with an "As Is" model using UML use case diagram, a classical tool for creating connections among users and stakeholders of a system. This model explains the system at high level. Although UML is widely used in the ICT area, its adoption in mechanical and industrial engineering sectors is still somehow limited. Therefore, more than pursuing advanced modeling aspects, this phase of the project was mostly concerned with the integration of these classical modeling techniques in the shop-floor engineering and assessing its use.

Fig 3 shows the main use cases of the system, which have interaction with relevant actors: Machining, Assembling, Quality Control, Execution System, Handling and, Maintenance. It requires three types of actors: Supervisor, Operator and Servicer.

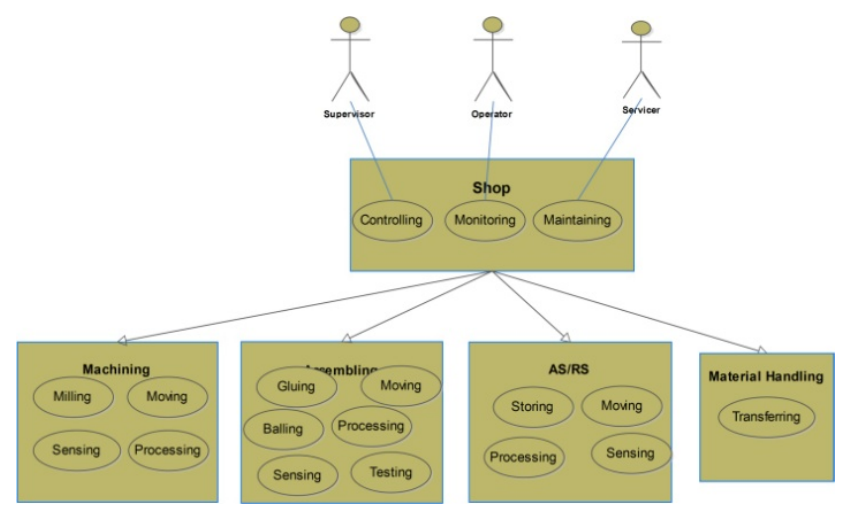

Fig. 3. Shop use case diagram

General Manufacturing Capability Model. The generic manufacturing capability model in the top-level diagram [3], [11] includes the main classes and their relationships. In this model, a facility comprises one or many resources, processes, and knowledge. Among these, there are associated relationships. The key role of the associations between classes shows that resources perform processes, and knowledge constrains either one or both resources and processes. Any event of a process is related to one or many instances of the resources features that specify the precondition and post-condition of that particular process. 
Each resource feature can relate to one or multiple different processes. Knowledge elements "regulate" the use of resources and processes.

Process Modeling. UML activity and sequence diagrams are used to represent the manufacturing processes of the shop-floor and its stations. Fig. 4 shows an example of activity diagram.

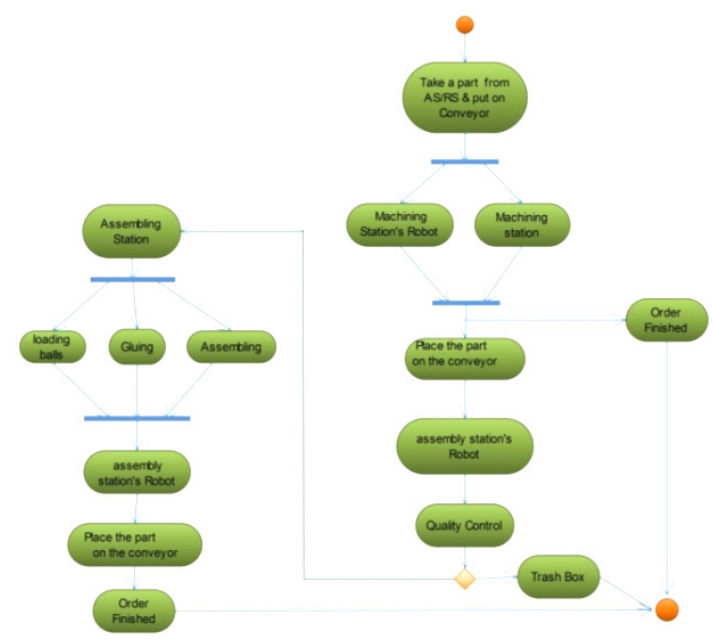

Fig. 4. Shop activity diagram

Fig. 5 shows a sequence diagram for the shop-floor case describing the levels of communication in the system. It represents the activities and processes related to each scenario, followed by the sequence of messages to be performed on each scenario depending on their need or specification. The rectangles represent a manufacturing process and each of the columns illustrates a manufacturing activity.

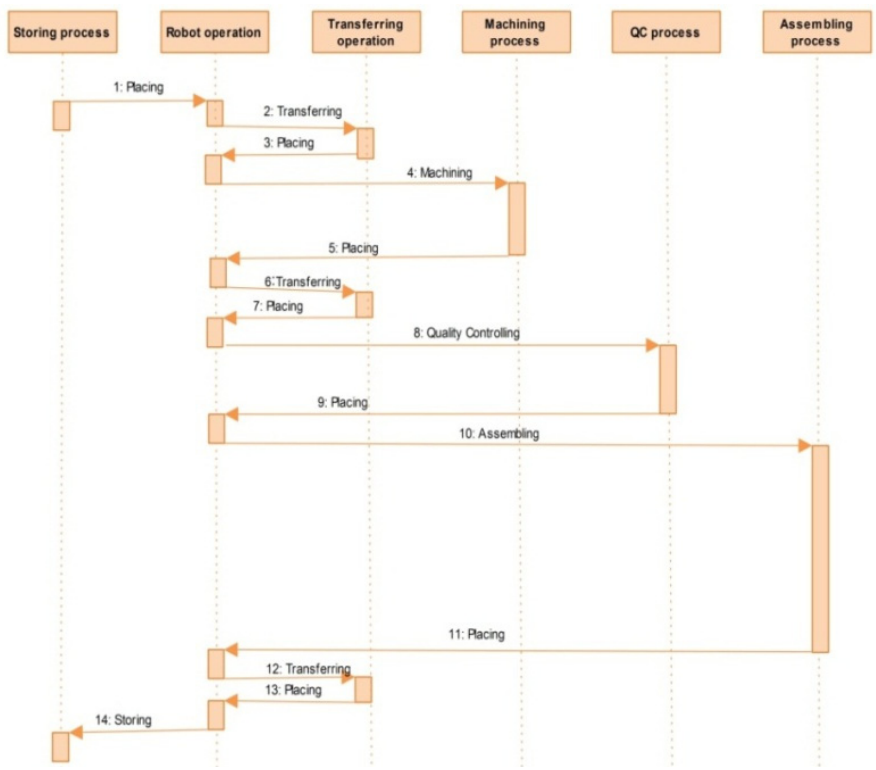

Fig. 5. Shop sequence Diagram 
Resources Modeling. UML object diagrams are used to represent "forward and reverse engineering" object relationships of a system, static view of an interaction, understanding object behavior and their relationships.

The object diagrams of the shop-floor involve the machining, assembling and AS/RS stations that realize the added value processes utilizing the station's resources. The material flow between the stations is realized by a conveyer. Meanwhile, data flow between stations is integrated using a host computer. Each of the stations uses several resources, with the material flow between the station's resources realized by a station's robot.

Type of Knowledge Modeling. Manufacturing knowledge is an important part of the intended capability-based manufacturing management system. Therefore, it is necessary to follow a structure that allows the access and storage of the wide range of manufacturing knowledge [11]. To define these knowledge structures it is necessary to explain what process and resource knowledge the manufacturing facility has and how they can be represented. Graphs, texts, tables, diagrams, formulas are some of the examples for explicit knowledge. While patterns, storytelling, video-clips and sketches are instances of tacit knowledge representation. Knowledge related to manufacturing processes and manufacturing resources are structured using different representation groups: explicit process knowledge, tacit process knowledge, implicit process knowledge, explicit resource knowledge, tacit resource knowledge and, implicit resource knowledge [12].

\section{Verification Block}

The verification block relies on the generation of data file using the results of "Preliminary study and problem definition phase" and then comparing the generated data file using the developed UML diagrams from the "Design and development phase" in order to understand whether the designed model meets the problem definition or not. XML is used to encode the exchange of information between the "Preliminary study, problem definition phase" and "Design and development phase" data files. The data file generated at the "Preliminary study and problem definition phase" consist of three groups of information:

1. Supporting data structures: (a) measurement units (time units); (b) statistics (standard deviations, average, state time, running time); (c) model references (model name, user, date, time,).

2. Manufacturing data structures: (a) element name; (b) element class; (c) resources (machines, labors, etc.); (d) operations lists; (e) events lists; (f) element failures; (g) daily schedules; (h) element busy time; (i) element idle time.

3. Objects negotiation data structures: (a) message name; (b) start message element; (c) end message element; (d) start messaging time; (e) end messaging time.

As the system's models are executed at "design and development phase", the UML diagrams are transformed into a new data file. After the consistency rules are applied to the both data files, the matching environment that captures the differences between 
two data files is ready for verification process. Discrepancies between the phases can be easily captured in the machining environment (i.e. the differences between the messages, events, operations, etc.).

\section{$7 \quad$ Implementation}

Transferring the Logical Model into a Data Base. JDeveloper is used to map the logical UML models into the Object Store $\subset$ data base.

Capability-Base Decision Support System. To support capability-based decision making processes, a Capability Analysis Tool (CAT) was developed. Fig. 6 illustrates the main components of CAT. Involved processes are briefly described below. The numbers in the figure stand for the index of each process.

- Step I: the order related component specifications (feature-based) are loaded into the "Capability Engine" to generate the required capabilities for the order (1).

- Step II: when the system wants to know availability of the required capabilities within the enterprise, the CA Tool is triggered and acquires capabilities which, newly generated on the previous steps are sent to the CA Tool (2).

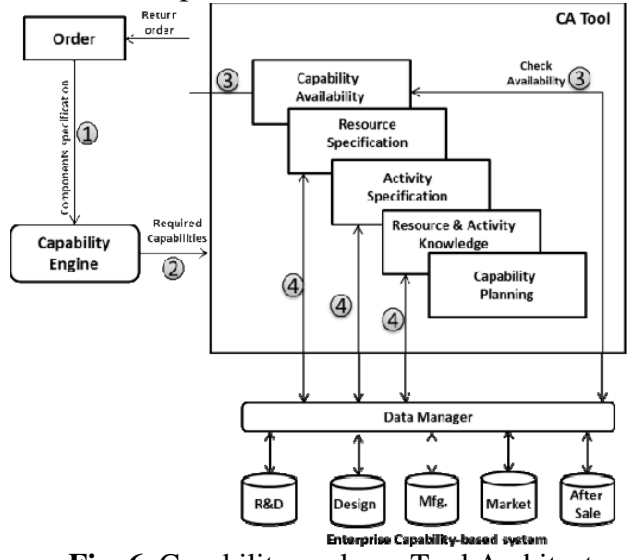

Fig. 6. Capability analyses Tool Architecture

- Step III: the CA Tool checks the enterprise capabilities and if the required capabilities are not available within the enterprise, the CA Tool returns a corresponding message to the order (3). This constitutes the base to identify the need for a collaborative partnership with other enterprises.

- Step IV: if the required capabilities are available in the enterprise, the information related to resources, activities and corresponding knowledge are obtained from the system (4). The CA Tool suggests appropriate capability plans for the required capabilities by invoking the information and knowledge of Step III.

- Step V: with the CA Tool results, the potential required information and knowledge for the order is highlighted and ready for the decision making process.

\section{Conclusion}

In this paper a capability-based decision support system for a manufacturing is structurally modeled using a case study of Computer Integrated Manufacturing laboratory of Eastern Mediterranean University. The research methodology of this 
contribution is divided into three phases, namely; "Preliminary study, problem definition phase", "Design and development phase", and "Implementation phase". An Object-oriented analysis approach is used as the modeling approach and UML used as modeling languages. A Capability Analysis Tool is developed as decision support systems for resource allocation and process and knowledge selection in case of a new business opportunity of the manufacturing system appears. An experimental system has been implemented using the object-oriented database Object Store $($ ) and the Visual $\mathrm{C}++$ programming environment. The developed experimental system offers four benefits, in that they a) enhance the organizations willingness to collaborate, $b$ ) boost the organization's competitiveness, c) facilitate appropriate decision-making, and d) finally help to integrate the entire organization.

\section{References}

1. Barenji, R.V., Hashemipour, M., Guerra-Zubiaga, D.A.: Toward a Modeling Framework for Organizational Competency. In: Camarinha-Matos, L.M., Tomic, S., Graça, P. (eds.) DoCEIS 2013. IFIP AICT, vol. 394, pp. 142-151. Springer, Heidelberg (2013)

2. Javidan, M.: Core competence: What does it mean in practice? Long Range Planning, 60-71 (1998)

3. Malhotra, Y.: Expert systems for knowledge management: crossing the chasm between information processing and sense making. Expert Systems with Applications, 7-16 (2001)

4. Guerra-Zubiaga, D.A., Young, R.I.M.: Design of a Manufacturing Knowledge Model. Int. Journal of Computer Integrated Manufacturing, 526-539 (2008)

5. Barenji, V.R., Hashemipour, M., Barenji, A.V., Guerra-Zubiaga, D.A.: Toward a framework for intra-enterprise competency modeling. In: 2nd Int. Conf. Advances in Computational Tools for Engineering Applications, pp. 284-288. IEEE explorer, Beirut (2012)

6. Kristianto, Y., Helo, P., Takala, J.: Manufacturing capabilities reconfiguration in manufacturing strategy for sustainable competitive advantage. International Journal of Operational Research, 82-101 (2011)

7. Molina, A., Ellis, T.I.A., Young, R.I.M., Bell, R.: Modeling manufcaturing capabilities to support concurrent engineering. Concurrent Engineering: Research and Applications (ACERA) Journal, 29-42 (1995)

8. Zhao, J., Cheung, W.M., Young, R.I.M.: A consistent manufacturing data model to support virtual enterprises. Int. Journal of Agile Management Systems, 150-158 (1999)

9. Liu, S., Young, R.I.M., Ding, L.: An integrated decision support system for global manufacturing co-ordination in the automotive industry. International Journal of Computer Integrated Manufacturing, 285-301 (2011)

10. Ho, W., Xu, X., Dey, P.K.: Multi-criteria decision making approaches for supplier evaluation and selection: a literature review. European J. Operational Research, 16-24 (2010)

11. Liu, S., Leat, M., Moizer, J., Megicks, P., Kasturiratne, D.: A decision-focused knowledge management framework to support collaborative decision making for lean supply chain management. I. J. Production Research, 2123-2137 (2013)

12. Guerra, D., Young, R.I.M.: Information and knowledge interrelationships within a manufacturing knowledge model. I. J. Adv Manufacturing Technology, 182-198 (2007) 